\title{
Self-Interfering Wave Packets
}

\author{
David Colas ${ }^{1}$ and Fabrice P. Laussy $y^{2,1, *}$ \\ ${ }^{1}$ Departamento de Física Teórica de la Materia Condensada and Condensed Matter Physics Center (IFIMAC), \\ Universidad Autónoma de Madrid, E-28049 Madrid, Spain \\ ${ }^{2}$ Russian Quantum Center, Novaya 100, 143025 Skolkovo, Moscow Region, Russia
}

(Received 11 July 2015; revised manuscript received 5 October 2015; published 13 January 2016)

\begin{abstract}
We study the propagation of noninteracting polariton wave packets. We show how two qualitatively different concepts of mass that arise from the peculiar polariton dispersion lead to a new type of particlelike object from noninteracting fields_-much like self-accelerating beams — shaped by the Rabi coupling out of Gaussian initial states. A divergence and change of sign of the diffusive mass results in a "mass wall" on which polariton wave packets bounce back. Together with the Rabi dynamics, this yields propagation of ultrafast subpackets and ordering of a spacetime crystal.
\end{abstract}

DOI: 10.1103/PhysRevLett.116.026401

Field theory unifies the concepts of waves and particles [1]. In quantum physics, this brought to rest the dispute of the pre-second-quantization era, on the nature of the wave function. As one highlight of this conundrum, the coherent state emerged as an attempt by Schrödinger to prove to Heisenberg that his equation is suitable to describe particles since some solutions exist that remain localized [2]. However, the reliance on an external potential and the lack of other particle properties-like resilience to collisions-makes this qualification a moot point and quantum particles are now understood as excitations of the field. The deep connection between fields and particles is not exclusively quantum and classical fields also provide a robust notion of particles, most famously with solitons [3]. The particle cohesion is here assured self-consistently by the interactions, allowing free propagation and surviving collisions with other solitons (possibly with a phase shift). For a long time, this has been the major example of how to define a particle out of a classical field, until Berry and Balázs (an assistant of Schrödinger himself) discovered the first case of a similar behavior in a noninteracting context: the Airy beams [4]. These solutions to the Schrödinger equation (or, equivalently, through the Eikonal approximation, to Maxwell equations) retain their shape as they propagate as a train of peaks (or subpackets) and also exhibit self-healing after passing through an obstacle [5]. The ingredient powering these particle behaviors is phase shaping, assuring the cohesion by the acceleration of the subpackets inside the mother packet. The solution was first regarded as a mathematical curiosity as it is not normalizable, until a truncated version was experimentally realized and shown to exhibit this dramatic phenomenology but for a finite time [6]. The Airy beam is now a recognized particlelike object, in some cases emerging from fields that quantize elementary particles [7], thus behaving like a metaparticle. It is in fact but one example of a full family of so-called "accelerating beams" [8], that all similarly endow linear fields with particle properties: shape preservation and resilience to collisions.

In this Letter, we add another member to the family of mechanisms that provide noninteracting fields with particle properties. Namely, we show that two coupled fields of different masses can support self-interfering wave packets, resulting in the propagation of a train of subpackets, much like the Airy beam, but without acceleration, fully normalizable and self-created out of a Gaussian initial state. Such coupled fields can be conveniently provided in the laboratory by polaritons [9], the superposition of light $\psi_{C}(x, t)$ and matter fields $\psi_{X}(x, t)$, cf. Fig. 1. They find their most versatile and tunable implementation in semiconductors where excitons (electron-hole pairs) of a quantum well are coupled to microcavity photons. Dispersions can furthermore be tuned by light-matter engineering, e.g., with photonic crystal polaritons [10]. Since polaritons can form condensates with a wave function describing their
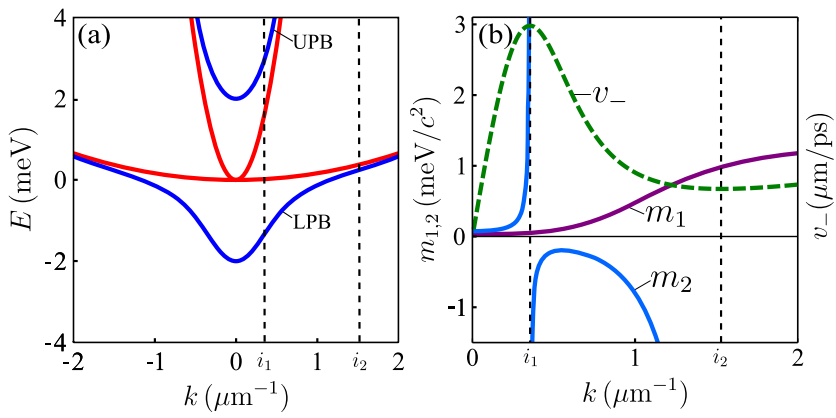

FIG. 1. (a) Polariton dispersions. In red: the parabolic dispersion of the cavity photon, and the bare exciton. In blue: the polariton branches $E_{ \pm}$. The vertical dashed lines at $i_{1}$ and $i_{2}$ mark the inflection points of the LPB. Parameters: $\Omega_{R}=2 \mathrm{meV}$, $m_{C}=0.025 \mathrm{meV} \mathrm{ps}^{2} \mu \mathrm{m}^{-2}, m_{X}=2 \mathrm{meV} \mathrm{ps}^{2} \mu \mathrm{m}^{-2}$. (b) Effective masses for the LPB as a function of $k$ : inertial mass $m_{1}$ (purple), diffusive mass $m_{2}$ (blue, negative when $i_{1}<k<i_{2}$ ), and group velocity $v_{-}$(green). 
collective dynamics [11], they are a dream laboratory to investigate the wave packet propagation in a variety of contexts [12], such as propagation of spin [13], bullets [14], or Rabi oscillations [15] with technological applications already in sight $[16,17]$. Polaritons are highly valued for their nonlinear properties due to particles' self-interactions [18], illustrated by a whole family of solitons (bright, dark, composite...) [19-22]. Recently, however, also the noninteracting regime has proved to be topical, with reports of Skyrmion analogues [23], band structure engineering [24] focusing and conical diffraction [25], Bosonic Josephson junctions [26], emulates of oblique dark and half solitons [27], topological edge modes [28,29], or the implementation of Hebbian learning in neural networks [30] to name a few but illustrative examples. In most of these cases, interactions bring the physics to even farther extents rather than spoiling the underlying linear effect, that remains nevertheless the one capturing the phenomenon. The linear regime can be achieved at low densities [31] since the polariton interaction at the few-particles level is small. The dynamics of the wave function $|\psi\rangle$ is then ruled by the polariton propagator $\Pi$ such that $|\psi(t)\rangle=\Pi\left(t-t_{0}\right)\left|\psi\left(t_{0}\right)\right\rangle$. In free space, it is diagonal in $k$ space [18]:

$$
\left\langle k^{\prime}|\Pi(t)| k\right\rangle=\exp \left[-i\left(\begin{array}{cc}
\frac{\hbar k^{2}}{2 m_{C}}+\Delta & \Omega_{R} \\
\Omega_{R} & \frac{\hbar k^{2}}{2 m_{X}}
\end{array}\right) t\right] \delta\left(k-k^{\prime}\right),
$$

where $m_{C,(X)}$ is the photon (exciton) mass, $\Delta$ their detuning, and $\Omega_{R}$ their Rabi coupling. The eigenstates of the propagator, $\left.\left.\Pi(t) \| k\rangle\rangle_{ \pm}=\exp \left(-i E_{ \pm} t\right) \| k\right\rangle\right\rangle_{ \pm}$, define both the polariton dispersion $E_{ \pm}=\hbar k^{2} m_{+}+2 \Delta \mp k_{\Omega}^{2}$ and the canonical polariton basis $\| k\rangle_{ \pm} \propto\left[E_{ \pm}(k), 1\right]^{T}|k\rangle$, where $m_{ \pm}=\left(m_{C} \pm m_{X}\right) /\left(m_{C} m_{X}\right)$ are the reduced relative masses, $k_{\Omega}=\sqrt[4]{\hbar^{2} k^{4} m_{-}^{2}-4 \hbar k^{2} \Delta m_{-}+4\left(\Delta^{2}+4 \Omega_{R}^{2}\right)}$ the dressed momentum and $|k\rangle$ the plane wave of well-defined momentum $k$. We use the notation ||$\rangle\rangle_{ \pm}$for upper $(+)$ and lower (-) polaritons. A general polariton state is thus expressed as $\left.\left.\| \psi\rangle\rangle_{ \pm}=\int_{-\infty}^{\infty} \phi_{ \pm}(k) \| k\right\rangle\right\rangle_{ \pm} d k$, where $\phi_{ \pm}(k)$ is the scalar-field polariton wave function. Except for a welldefined polariton state in $k$ space, i.e., a fully delocalized polariton in real space, the photon and exciton components of a polariton cannot be jointly defined by a given wave packet $\phi(k)$. Indeed, except if $\phi(k)=\delta(k)$, one component gets modulated by the $E_{ \pm}(k)$ factor needed to maintain the particle on its branch. One consequence of this composite structure is that a polariton cannot be localized in real space, with both its photon and exciton components simultaneously localized. Choosing $\phi(k)$ such that either $\psi_{C}(x, t=0)$ or $\psi_{X}(x, t=0)$ is $\delta(x)$ smears out the other component around the localized field, as shown in Figs. 2(a)-2(b). Such constraints result in a rich phenomenology with a large enough set of momenta. We now discuss some of the effects that arise from self-shaping and self-interferences of polaritons due to their composite structure.
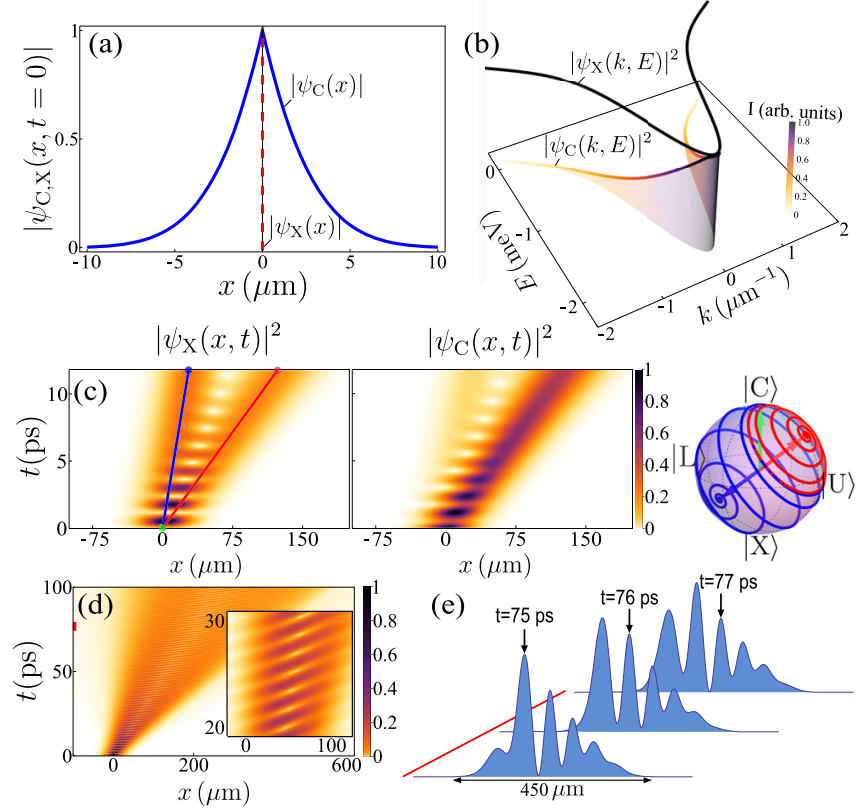

FIG. 2. (a) Localizing a polariton in space is possible for one of its component only (here in dashed red); the other field smears out to keep the particle on its branch. (b) Counterpart of (a) in energymomentum space. (c) Spacetime evolution of $\left|\psi_{C}(x, t)\right|^{2}$ and $\left|\psi_{X}(x, t)\right|^{2}$ with a photon of momentum $k_{0}=0.5 / \mu \mathrm{m}$ as an initial condition, with quantum states along the lines shown on the Bloch sphere. (d) Configuration with $\Delta=-\Omega_{R}$, preventing splitting of the beam and resulting in ultrafast, Rabi-powered, propagating subpackets, as shown for three snapshots of time in (e).

It has long been known that the mass imbalance $m_{C} \ll m_{X}$ results in peculiar dispersions for the upper $\left(E_{+}\right)$and lower $\left(E_{-}\right)$polariton branches, shown in Fig. 1(a) along with the parabolic dispersions of the light photon and the heavy exciton, meeting at $k=0(\Delta=0)$. In order to provide a comprehensive picture including the two inflection points that can result for the lower polariton branch (LPB), we assume here a smaller mass ratio than in typical experiments. The most important effects are, however, due to the first inflection point that is found in most samples. To access more inflection points, one can also turn to other platforms such as modulated photonic lattices [32], crystals [10], or coupled wave guides [33] (we refer to the Supplemental Material [34] for further discussion and experimental implementations with currently available systems). The dynamics of a Gaussian wave packet large enough in space to probe only parabolic portions of the dispersion in reciprocal space is essentially that expected from Schrödinger dynamics [35], diffusing with mean standard deviation of the packet size [34]:

$$
\sigma_{x}(t)=\sqrt{\sigma_{x}^{2}(0)+\left(\hbar t /\left[2 m_{2} \sigma_{x}(0)\right]\right)^{2}} .
$$

For nonparabolic dispersions, the degeneracy is lifted for some of the various concepts of masses, famously unified for the gravitational and inertial masses by Einstein as part 
of his theory of gravitation. Wave packets have two different effective masses, $m_{1}$ and $m_{2}$ [36], describing, respectively, propagation and diffusion. A wave packet propagates with a group velocity $v_{ \pm}=\partial_{k} E_{ \pm}(k)$. This defines the inertial mass $m_{1}$ that determines the wave packet velocity from de Broglie's relation $p=\hbar k$ and the classical momentum $p=m v_{ \pm}$as

$$
m_{1}(E, k)=\hbar^{2} k\left(\partial_{k} E\right)^{-1} .
$$

A second mass $m_{2}$, that we will call the diffusive mass, is associated with the spreading of the wave packet according to Eq. (2) and depends on the branch's curvature; it reads

$$
m_{2}(E, k)=\hbar^{2}\left(\partial_{k}^{2} E\right)^{-1} .
$$

These two masses $m_{1}, m_{2}$, and the packet velocity $v_{-}$are plotted in Fig. 1(b) for the LPB. Unlike parabolic dispersions, where they are equal, polariton dispersions yield different inertial and diffusive masses. In particular, the $k$-dependent inertial mass $m_{1}$ imposes a maximum speed for the lower polaritons [34]. Beyond the inflection point $i_{1}$, polaritons slow down with increasing momentum (at large $k$, polaritons become bare particles with no such kinematic restrictions). The coupling of the two fields with different masses results in the heavier one lagging behind the other, as seen in Fig. 2(c) where a Gaussian photon wave packet is imparted with a momentum $k=0.5 / \mu \mathrm{m}$, achieved experimentally by sending a pulse at an angle and overlapping both branches. This prevents the photon and exciton packets to propagate Rabi oscillating, and instead forces a splitting in two beams-the orthogonal polariton states which are eigenstates for the corresponding wave vector, as shown by their trajectory on the Bloch sphere-connected by a Rabi oscillating tunnel. The Rabi oscillations only take place when there is a spatial overlap between the polaritons. The two propagating packets maintain their coherence despite their space separation and would Rabi oscillate if meeting again, due, for instance, to a ping-pong reflection [37]. The splitting in two beams can be minimized by tuning parameters to equalize the polaritons masses, in particular, the inertial ones. Combined with the bending of the Rabi oscillations in spacetime, which can be achieved at nonzero detuning, this leads to propagation of Rabi oscillations, which produce ultrafast subpackets moving inside a mother packet, as shown in Fig. 2(d) and for three time snapshots in 2(e). The subpackets, continuously formed in the tail of the mother packet, propagate inside 1 order of magnitude faster, powered by Rabi oscillations, before dying in the head. Each subpeak acquires properties of an identifiable object, traceable in time. The full dynamics is available in an accompanying video [34]. Now on the diffusive mass $m_{2}$ : it diverges at the two inflection points $i_{1,2}$ of the LPB and is negative in between. Exciting at the inflection points thus cancels diffusion as seen in Eq. (2) and in Figs. 3(a) and 3(b) with the propagation of a broad $\left[\sigma_{x}(0)=20 \mu \mathrm{m}\right]$ lower-polariton wave packet with an imparted momentum of (a) $k_{0}=0$ and (b) $k_{0}=i_{1}$. The excitation around the inflection point has already been used to generate bright solitons and soliton trains $[19,20,38,39]$. In these cases, the soliton mechanism is the interplay between negative effective mass and repulsive interactions. The role of the high effective mass close to the inflection point, which cancels the diffusion, was not, however, fully estimated.

The interesting phenomenology discussed so far illustrates isolated features of polariton propagation. A new physical picture emerges when combining several aspects within the same wave function, leading to the concept of
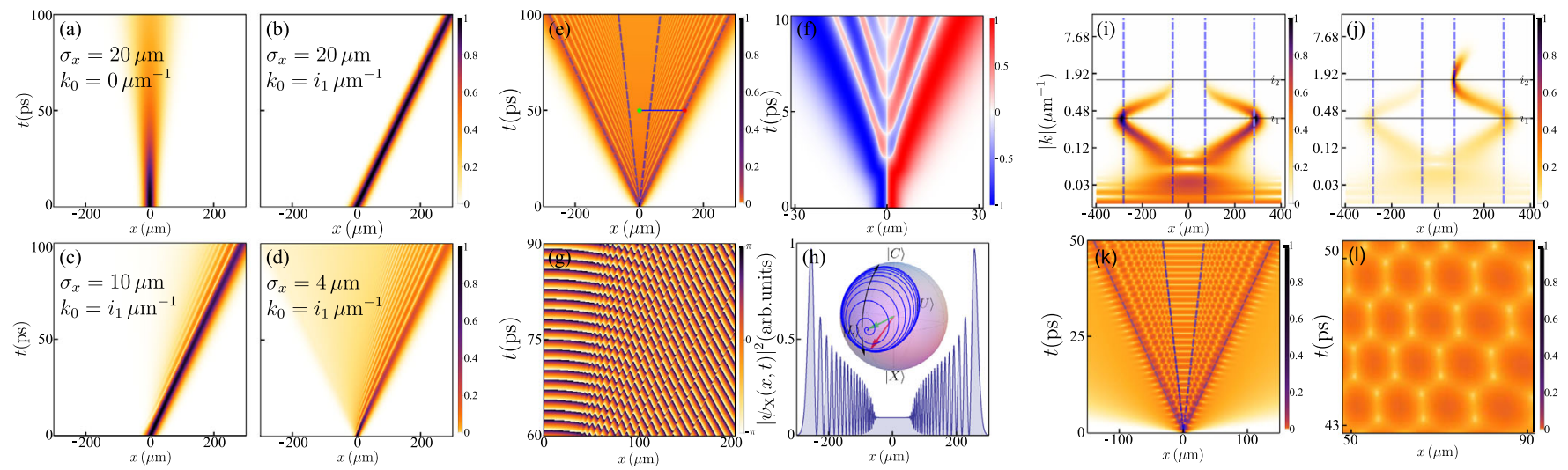

FIG. 3. (a)-(d) Propagation of lower polariton packets for various momenta and size, showing the emergence of the SIP for narrower packets. (e) Dynamics of an even narrower packet $\left[\sigma_{x}(0)=2 \mu \mathrm{m}\right.$ with no momentum $\left.k_{0}=0\right]$. (f) Current probability $j$ at early times, showing the coexistence and interleaving of net counterpropagating flows. (g) Phase map in a selected region, showing $\pi$ jumps associated to each subpeak. (h) Intensity profile at $t=90 \mathrm{ps}$ with the evolution of the quantum state on the Bloch sphere corresponding to the path (from green to red) plotted in (e). (i) Wavelet decomposition of (e) at $t=100 \mathrm{ps,} \mathrm{and} \mathrm{(j)} \mathrm{in} \mathrm{the} \mathrm{same} \mathrm{configuration} \mathrm{but}$ exciting with a momentum $k_{0}=i_{2}$. (k) Spacetime honeycomb lattice when combining the SIP with Rabi oscillations by starting with a photon as an initial condition. (1) Zoom of the hexagonal lattice. 
self-interfering packets (SIP). This occurs when reducing the wave packet size in real space, that is, increasing the staggering on the dispersion in momentum-space $\left[\sigma_{k}(0)=1 / \sigma_{x}(0)\right]$, to an extent enough to probe polaritonic deviation from the parabolic dispersion. In this case, the negative mass plays an explicit role. Negative masses are a recurrent theme in physics but this is typically meant for the inertial mass [40]. The sign of the diffusive mass would seem not to play a role since it is squared in Eq. (2), and this is indeed the case for momenta $i_{1}<k<i_{2}$. When straddling over the divergence, however, self-interferences occur between harmonics of the packet subject to the positive mass and others to the negative mass. This results in a reshaping of the wave function, as shown in Figs. 3(c) and $3(\mathrm{~d})$ decreasing $\sigma_{x}(0)$ down to 10 and $4 \mu \mathrm{m}$. The part of the packet that goes beyond the divergence is reflected back and interferes with the rest that still propagates forward, resulting in ripples. Reducing the packet to $\sigma_{x}(0)=2 \mu \mathrm{m}$ produces the striking pattern seen in Fig. 3(e), without even imparting momentum. While for a parabolic dispersion, squeezing the packet in space merely causes a faster diffusion, in the polariton case, there is thus a critical diffusion beyond which the packet stops expanding and folds back onto itself. Since this happens when the wave function encounters the inflection point of the LPB, there is a "mass wall" against which the packet bounces back. If the dispersion also features another inflection point at larger $k$, this reflection happens again, shielding the core of the mother packet from this selfinterference, as shown on the cut in intensity in Fig. 3(h) [the diffusion cones are the solution of $\partial_{k}^{2} E_{-}=0$, cf. Fig. 3(e)]. More importantly from a conceptual point of view, as a result of this coexistence of masses of opposite signs within the same packet, the mother wave packet $|\psi\rangle$ fragments itself into two trains of daughter shapepreserving subpackets traveling in opposite directions. The overall momentum $\langle\psi|p| \psi\rangle=0$ is null but the selfshaping of the wave function redistributes it through its subpackets with nonzero momenta. Each subpeak can be identified as a polariton lying onto the meridian between $|L\rangle$ and $|X\rangle$, as seen by following its quantum state on the Bloch sphere, Fig. 3(h). The SIP can therefore be seen as a train of successive polariton packets, "emitted" at the rate of Rabi oscillations by the area shielded from selfinterferences, retaining their individuality as they propagate inside the mother packet. The quantum state dynamics along these paths is seen vividly in a video in the Supplemental Material [34]. Successive peaks furthermore feature a maximal phase shift of $\pi$ in the phase $\phi(x, t)$ of the total wave function $\psi(x, t)=|\psi(x, t)| \exp [i \phi(x, t)]$, as shown in Fig. 3(g). Although they do not involve self-interactions to account for their cohesion, these propagating subpackets behave in many respects as solitonlike objects. The analogy with Airy beams is conspicuous.
One can gain additional insights into the nature of the SIP through the current probability $j=i \hbar / 2 m_{1}\left(\psi^{*} \partial_{x} \psi-\right.$ $\left.\psi \partial_{x} \psi^{*}\right)$, cf. Fig. 3(f), where the packet is plainly seen to alternate backward and forward net flows. Alternatively, considering the wavelet transform (WT) [41] $\mathbb{W}_{a, b}(\psi)=$ $(1 / \sqrt{|a|}) \int_{-\infty}^{+\infty} \psi(x) \mathcal{G}^{*}[(x-b) / a] \mathrm{d} x$, in our case, of the Gabor wavelet family $\mathcal{G}(z)=\sqrt[4]{\pi} \exp (i \omega x) \exp \left(-x^{2} / 2\right)$, allows us to decompose the wave function into Gaussian packets, which are the basic packets as far as propagation and diffusion are concerned. This extension of the Fourier transform is common in signal processing but has found so far little echo to study wave packet dynamics [42]. We show in Fig. 3(i) the energy density $\left|\mathbb{W}_{k, x}\right|^{2}$ of the wave function in the $(x-k)$ plane at $t=100 \mathrm{ps}$. One can see clearly how self-interferences confine the polariton packet within the diffusion cone (blue dashed lines) by diverting the flow backward, (i) one or (j) two times when the second inflection point $i_{2}$ is reached. Other fundamental connections can be established. For instance, patterns strikingly similar to Fig. 3(e) were observed in the quenched dynamics of a quantum spin chain with magnons [43], a completely different system. This suggests that coupled light fields feature fundamental and universal dynamical evolutions. Combining this characteristic pattern with that of Rabi oscillations leads to the spacetime propagation presented in Fig. 3(k). The protected area exhibits simple Rabi oscillations. The outer area is propagating upper polaritons and is not affected either by oscillations nor interferences. In the SIP area, however, sitting between the two mass walls, the interplay of Rabi oscillations and self-interferences produces a hexagonal lattice. Such a structure is known to arise from interferences of three beams $[44,45]$ and indeed in our case, it arises from interferences of two LPs (on both sides of the inflection point) and one UP [34]. This remarkable structure is, again, sculpted self-consistently out of a unique photon field with a simple Gaussian shape by the dynamics of coupled noninteracting fields. Here, instead of the emergence of propagating particles, a spacetime crystal is formed with the manifest ordering of the previously freely propagating train of polaritons.

In conclusions, we discussed the intricate wave packet propagation of coupled fields (polaritons). Unlike the eventual complete indeterminacy of Schrödinger wave packets in a parabolic dispersion, polaritons can sustain traceable objects with always well-defined properties, such as their shape, position, momentum, and quantum state. This gives rise to a concept of particles similar to that brought by solitons in nonlinear media or Airy beams in noninteracting ones. While these are formed by selfinteraction and phase shaping, the individuality of polaritons is acquired and maintained through self-interferences powered by the Rabi coupling. This shows that even in the linear regime, the polariton dynamics is rich and able to produce complex structures out of mere Gaussian initial states. This could lead to applications, following the spirit 
of self-accelerating beams. For instance in the classical regime, in a way similar to particle clearing through Airy wave packets [46], polaritons could impart momentum powered by the Rabi oscillations, or, by exciting polaritons with quantum light [47], quantum SIP could propagate in properly wired polariton circuits to perform Linear optical quantum computing, thanks to the linearity of the effect. SIP can indeed carry qubit states at the one-particle level, unlike solitons with which they otherwise share similar propagation qualities.

This work is funded by the "POLAFLOW" ERC Project No. 308136. We thank L. Dominici, D. Ballarini, E. del Valle, and D. Sanvitto for discussions.

* Corresponding author.

fabrice.laussy@gmail.com

[1] T.-K. Ng, Introduction to Classical and Quantum Field Theory (Wiley, New York, 2009).

[2] E. Schrödinger, Der stetige übergang von der Mikro- zur Makromechanik, Naturwissenschaften 14, 664 (1926).

[3] D. J. Korteweg and G. de Vries, On the change of form of long waves advancing in a rectangular canal, and on a new type of long stationary waves, Philos. Mag. B 39, 422 (1895).

[4] M. V. Berry and N. L. Balazs, Nonspreading wave packets, Am. J. Phys. 47, 264 (1979).

[5] J. Broky, G. A. Siviloglou, A. Dogariu, and D. N. Christodoulides, Self-healing properties of optical Airy beams, Opt. Express 16, 12880 (2008).

[6] G. A. Siviloglou, J. Broky, A. Dogariu, and D. N. Christodoulides, Observation of Accelerating Airy Beams, Phys. Rev. Lett. 99, 213901 (2007).

[7] N. Voloch-Bloch, Y. Lereah, Y. Lilach, A. Gover, and A. Arie, Generation of Electron Airy beams, Nature (London) 494, 331 (2013).

[8] P. Zhang, Y. Hu, T. Li, D. Cannan, X. Yin, R. Morandotti, Z. Chen, and X. Zhang, Nonparaxial Mathieu and Weber Accelerating Beams, Phys. Rev. Lett. 109, 193901 (2012).

[9] A. Kavokin, J. J. Baumberg, G. Malpuech, and F. P. Laussy, Microcavities, 2nd ed. (Oxford University Press, New York, 2011).

[10] D. Gerace and L. C. Andreani, Quantum theory of excitonphoton coupling in photonic crystal slabs with embedded quantum wells, Phys. Rev. B 75, 235325 (2007).

[11] J. Kasprzak et al., Bose-Einstein condensation of exciton polaritons, Nature (London) 443, 409 (2006).

[12] D. Sanvitto, A. Amo, F. P. Laussy, A. Lemaître, J. Bloch, C. Tejedor, and L. Viña, Polariton condensates put in motion, Nanotechnology 21, 134025 (2010).

[13] I. A. Shelykh, Y. G. Rubo, G. Malpuech, D. D. Solnyshkov, and A. Kavokin, Polarization and Propagation of Polariton Condensates, Phys. Rev. Lett. 97, 066402 (2006).

[14] A. Amo et al., Collective fluid dynamics of a polariton condensate in a semiconductor microcavity, Nature (London) 457, 291 (2009).
[15] T. C. H. Liew, Y. G. Rubo, and A. V. Kavokin, Excitonpolariton oscillations in real space, Phys. Rev. B 90, 245309 (2014).

[16] D. Ballarini, M. De Giorgi, E. Cancellieri, R. Houdré, E. Giacobino, R. Cingolani, A. Bramati, G. Gigli, and D. Sanvitto, All-optical polariton transistor, Nat. Commun. 4, 1778 (2013).

[17] T. Espinosa-Ortega and T. C. H. Liew, Complete architecture of integrated photonic circuits based on AND and NOT logic gates of exciton polaritons in semiconductor microcavities, Phys. Rev. B 87, 195305 (2013).

[18] I. Carusotto and C. Ciuti, Quantum fluids of light, Rev. Mod. Phys. 85, 299 (2013).

[19] O. A. Egorov, D. V. Skryabin, A. V. Yulin, and F. Lederer, Bright Cavity Polariton Solitons, Phys. Rev. Lett. 102, 153904 (2009).

[20] M. Sich, D. N. Krizhanovskii, M. S. Skolnick, A. V. Gorbach, R. Hartley, D. V. Skryabin, E. A. Cerda-Méndez, K. Biermann, R. Hey, and P. V. Santos, Observation of bright polariton solitons in a semiconductor microcavity, Nat. Photonics 6, 50 (2012).

[21] H. Flayac, D. D. Solnyshkov, and G. Malpuech, Oblique half-solitons and their generation in exciton-polariton condensates, Phys. Rev. B 83, 193305 (2011).

[22] G. Christmann, G. Tosi, N. G. Berloff, P. Tsotsis, P. S Eldridge, Z. Hatzopoulos, P. G. Savvidis, and J. J. Baumberg, Oscillatory solitons and time-resolved phase locking of two polariton condensates, New J. Phys. 16, 103039 (2014).

[23] D. V. Vishnevsky, H. Flayac, A. V. Nalitov, D. D. Solnyshkov, N. A. Gippius, and G. Malpuech, Skyrmion Formation and Optical Spin-Hall Effect in an Expanding Coherent Cloud of Indirect Excitons, Phys. Rev. Lett. 110, 246404 (2013).

[24] T. Jacqmin, I. Carusotto, I. Sagnes, M. Abbarchi, D. D. Solnyshkov, G. Malpuech, E. Galopin, A. Lemaître, J. Bloch, and A. Amo, Direct Observation of Dirac Cones and a Flatband in a Honeycomb Lattice for Polaritons, Phys. Rev. Lett. 112, 116402 (2014).

[25] H. Terças, H. Flayac, D. Solnyshkov, and G. Malpuech, Non-Abelian Gauge Fields in Photonic Cavities and Photonic Superfluids, Phys. Rev. Lett. 112, 066402 (2014).

[26] N. S. Voronova, A. A. Elistratov, and Y. E. Lozovik, DetuningControlled Internal Oscillations in an Exciton-Polariton Condensate, Phys. Rev. Lett. 115, 186402 (2015).

[27] P. Cilibrizzi, H. Ohadi, T. Ostatnicky, A. Askitopoulos, W. Langbein, and P. Lagoudakis, Linear Wave Dynamics Explains Observations Attributed to Dark Solitons in a Polariton Quantum Fluid, Phys. Rev. Lett. 113, 103901 (2014).

[28] A. Nalitov, D. Solnyshkov, and G. Malpuech, Polariton $\mathbb{Z}$ Topological Insulator, Phys. Rev. Lett. 114, 116401 (2015).

[29] T. Karzig, C.-E. Bardyn, N. H. Lindner, and G. Refael, Topological polaritons, Phys. Rev. X 5, 031001 (2015).

[30] T. Espinosa-Ortega and T. Liew, Perceptrons with Hebbian Learning Based on Wave Ensembles in Spatially Patterned Potentials, Phys. Rev. Lett. 114, 118101 (2015).

[31] L. Dominici et al., Ultrafast Control and Rabi Oscillations of Polaritons, Phys. Rev. Lett. 113, 226401 (2014). 
[32] I. L. Garanovich, S. Longhi, A. A. Sukhorukova, and Y. S. Kivshar, Light propagation and localization in modulated photonic lattices and waveguides, Phys. Rep. 518, 1 (2012).

[33] S. C. Huang, M. Kato, E. Kuramochi, C. P. Lee, and M. Notomi, Time-domain and spectral-domain investigation of inflection-point slow-light modes in photonic crystal coupled waveguides, Opt. Express 15, 3543 (2007).

[34] See Supplemental Material at http://link.aps.org/ supplemental/10.1103/PhysRevLett.116.026401 for additional analytical and numerical results on polariton propagation, possible implementations in realistic and dissipative systems, 2D cases and descriptions of movies.

[35] G. I. Márk, Analysis of the spreading Gaussian wavepacket, Eur. Phys. J. direct B 18, 247 (1997).

[36] J. Larson, J. Salo, and S. Stenholm, Effective mass in cavity QED, Phys. Rev. A 72, 013814 (2005).

[37] C. Antón, T. C. H. Liew, J. Cuadra, M. D. Martín, P. S. Eldridge, Z. Hatzopoulos, G. Stavrinidis, P. G. Savvidis, and L. Viña, Quantum reflections and shunting of polariton condensate wave trains: Implementation of a logic AND gate, Phys. Rev. B 88, 245307 (2013).

[38] O. Egorov, A. Gorbach, F. Lederer, and D. Skryabin, Two-Dimensional Localization of Exciton Polaritons in Microcavities, Phys. Rev. Lett. 105, 073903 (2010).

[39] M. Sich et al., Effects of Spin-Dependent Interactions on Polarization of Bright Polariton Solitons, Phys. Rev. Lett. 112, 046403 (2014).
[40] M. Wimmer, A. Regensburger, C. Bersch, M.-A. Miri, S. Batz, G. Onishchukov, D. N. Christodoulides, and U. Peschel, Optical diametric drive acceleration through action-reaction symmetry breaking, Nat. Phys. 9, 780 (2013).

[41] L. Debnath and F. A. Shah, Wavelet Transforms and Their Applications, 2nd ed. (Birkhăuser, Springer, Basel, 2015).

[42] C. H. Baker, D. A. Jordan, and P. M. Norris, Application of the wavelet transform to nanoscale thermal transport, Phys. Rev. B 86, 104306 (2012).

[43] W. Liu and N. Andrei, Quench Dynamics of the Anisotropic Heisenberg Model, Phys. Rev. Lett. 112, 257204 (2014).

[44] T. C. H. Liew, Y. G. Rubo, and A. V. Kavokin, Generation and Dynamics of Vortex Lattices in Coherent ExcitonPolariton Fields, Phys. Rev. Lett. 101, 187401 (2008).

[45] C. Becker, P. Soltan-Panahi, J. Kronjäger, S. Dörscher, K. Bongs, and K. Sengstock, Ultracold quantum gases in triangular optical lattices, New J. Phys. 12, 065025 (2010).

[46] J. Baumgartl, M. Mazilu, and K. Dholakia, Optically mediated particle clearing using Airy wavepackets, Nat. Photonics 2, 675 (2008).

[47] J. C. López Carreño, C. Sánchez Muñoz, D. Sanvitto, E. del Valle, and F. Laussy, Exciting Polaritons with Quantum Light, Phys. Rev. Lett. 115, 196402 (2015). 